\section{(2) OPEN ACCESS}

\title{
Long-term structural and symptomatic effects of intra-articular sprifermin in patients with knee osteoarthritis: 5-year results from the FORWARD study
}

\author{
Felix Eckstein (D) , 1,2,3 Marc C Hochberg, ${ }^{4}$ Hans Guehring, ${ }^{5}$ Flavie Moreau, ${ }^{6}$ \\ Victor Ona, ${ }^{6}$ Asger Reinstrup Bihlet, ${ }^{7}$ Inger Byrjalsen, ${ }^{7}$ Jeppe Ragnar Andersen, ${ }^{7}$ \\ Benjamin Daelken, ${ }^{5}$ Oliver Guenther, ${ }^{5}$ Christoph Ladel, ${ }^{5}$ Martin Michaelis, ${ }^{5}$ \\ Philip G Conaghan (D) ${ }^{8}$
}

\begin{abstract}
Handling editor Josef S Smolen

- Additional supplemental material is published online only. To view, please visit the journal online (http://dx.doi. org/10.1136/annrheumdis2020-219181).
\end{abstract}

For numbered affiliations see end of article.

\section{Correspondence to} Professor Marc C Hochberg, University of Maryland School of Medicine, Baltimore, Maryland USA;

mhochber@som.umaryland.edu

Received 25 September 2020 Revised 12 March 2021 Accepted 28 March 2021 Published Online First 7 May 2021

\section{Check for updates}

(C) Author(s) (or their employer(s)) 2021. Re-use permitted under CC BY-NC. No commercial re-use. See rights and permissions. Published by BMJ.

To cite: Eckstein $\mathrm{F}$ Hochberg MC, Guehring $\mathrm{H}$, et al. Ann Rheum Dis 2021;80:1062-1069.

\section{ABSTRACT}

Objective The FORWARD (FGF-18 Osteoarthritis Randomized Trial with Administration of Repeated Doses) trial assessed efficacy and safety of the potential diseasemodifying osteoarthritis drug (DMOAD) sprifermin in patients with knee osteoarthritis. Here, we report 5-year efficacy and safety results.

Methods Patients were randomised to intra-articular sprifermin $100 \mu \mathrm{g}$ or $30 \mu \mathrm{g}$ every 6 months (q6mo) or 12 months, or placebo, for 18 months. The primary analysis was at year 2, with follow-up at years 3, 4 and 5 . Additional post hoc exploratory analyses were conducted in patients with baseline minimum radiographic joint space width 1.5-3.5 $\mathrm{mm}$ and Western Ontario and McMaster Universities Osteoarthritis Index (WOMAC) pain 40-90, a subgroup at risk (SAR) of progression. Results 378 (69\%) patients completed the 5year follow-up. A significant dose-response in total femorotibial joint cartilage thickness with sprifermin (trend test, $\mathrm{p}<0.001$ ) and a $0.05 \mathrm{~mm}$ mean difference with sprifermin $100 \mu \mathrm{g}$ q6mo versus placebo $(95 \% \mathrm{Cl}$ 0.00 to $0.10 ; p=0.015$ ) were sustained to year 5 . WOMAC pain scores improved $\sim 50 \%$ from baseline in all groups. No patient in the $100 \mu \mathrm{g}$ q6mo group had replacement of the treated knee. $96 \%-98 \%$ of patients receiving sprifermin and $98 \%$ placebo reported adverse events, most were mild or moderate and deemed unrelated to treatment. Adverse event-related study withdrawals were $<10 \%$. Differentiation in WOMAC pain between sprifermin $100 \mu \mathrm{g} \mathrm{q6mo}$ and placebo in the SAR $(n=161)$ at year 3 was maintained to year $5(-10.08$; $95 \% \mathrm{Cl}-25.68$ to 5.53$)$.

Conclusion In the longest DMOAD trial reported to date, sprifermin maintained long-term structural modification of articular cartilage over 3.5 years posttreatment. Potential translation to clinical benefit was observed in the SAR.

Trial registration number NCT01919164

\section{INTRODUCTION}

Current therapies for osteoarthritis (OA) focus on reducing pain, improving physical function and patient's quality of life. ${ }^{12}$ There is an urgent need for disease-modifying osteoarthritis drugs (DMOADs) that improve symptoms and inhibit

\section{Key messages}

What is already known about this subject?

- Sprifermin is a potential disease-modifying osteoarthritis drug that was shown to increase articular cartilage thickness compared with placebo in patients with knee osteoarthritis at the primary endpoint (year 2) of the FORWARD trial and was well tolerated. Structural benefits with sprifermin were observed in a subgroup at risk (SAR) of progression, who also demonstrated improvements in pain over placebo at year 3 follow-up.

What does this study add?

- Here we show that long-term structural modification of articular cartilage was maintained with sprifermin versus placebo over a 3.5-year to 4-year post-treatment period. Similar maintenance of structural improvements over this time was seen in the SAR, and clinically relevant improvement in pain over placebo in this subgroup was also sustained up to year 5 follow-up.

- There were no target knee replacements in the highest dose group of sprifermin over 5 years and no new safety signals.

How might this impact on clinical practice or future developments?

- These findings confirm the long-term structural benefit of intra-articular sprifermin treatment, with potential long-term clinical benefit and disease modification for knee osteoarthritis and no safety concerns.

- Long-term follow-up in disease-modifying osteoarthritis drug trials may be required to ensure that translation of structural modification to clinical benefit is seen.

structural disease progression. ${ }^{3}{ }^{4}$ Many DMOAD trials have had limited success or yielded negative results, with the heterogeneous nature of OA and insufficient trial follow-up times being potential contributors. $^{56}$ 
Preservation of cartilage integrity is widely considered a highly important goal for a DMOAD. ${ }^{7}$ Sprifermin is a recombinant human fibroblast growth factor 18 that has cartilage regenerative properties and is under investigation as a potential DMOAD for knee OA. ${ }^{8-11}$ No measurable systemic effects or safety concerns were associated with sprifermin in phase I and Ib studies. ${ }^{12} 13$

FORWARD (FGF-18 Osteoarthritis Randomized Trial with Administration of Repeated Doses) was a 5-year, randomised clinical trial that evaluated the efficacy and safety of sprifermin among 549 patients with symptomatic radiographic knee OA. ${ }^{14}$ FORWARD was designed with total femorotibial joint (TFTJ) cartilage thickness change as the primary structural endpoint at year 2, and change in Western Ontario and McMaster Universities Osteoarthritis Index (WOMAC) scores over the same period was a secondary endpoint. Follow-up continued up to year 5 . The 2-year and 3-year data were reported recently: dosedependent modification of TFTJ cartilage thickness with sprifermin was demonstrated at years 2 and 3, and a mean difference of $0.05 \mathrm{~mm}$ in TFTJ cartilage thickness between the highest sprifermin dose (100 $\mu$ g every 6 months (q6mo); $0.03 \mathrm{~mm}$ gain) and placebo $\left(0.02 \mathrm{~mm}\right.$ loss) was observed. ${ }^{14}$ WOMAC total scores improved by around 50\% in all cohorts but did not differ significantly between sprifermin and placebo. ${ }^{14}$

Previous studies have revealed that including patients with at least moderate pain at baseline enables greater differentiation of a pain response to treatment. ${ }^{7}$ In addition, inclusion of patients with advanced disease (eg, low radiographic joint space width (JSW)) may enhance the ability to observe disease progression $^{15}$ and detect translation of structural modification to clinical benefit. ${ }^{16}$ Exploratory post hoc analyses were therefore conducted in a so-called 'subgroup at risk' (SAR) of patients in FORWARD, with narrower medial or lateral minimum JSW (mJSW) and higher WOMAC pain than the overall study population at baseline $(\mathrm{n}=161) .{ }^{16}$ SAR placebo-treated patients had more rapid structural progression compared with the overall study population, whereas net TFTJ cartilage modification with sprifermin versus placebo was similar. ${ }^{16}$ Importantly, in the SAR, differentiation in WOMAC pain score change between placebo and the highest sprifermin dose was observed at year $3 .^{16}$

Here, we report the long-term structural and symptomatic efficacy and safety of sprifermin in the overall FORWARD study population and SAR up to year 5, that is 3.5 (for the q6mo groups) or 4 years (for the q12mo groups) after the last sprifermin treatment.

\section{PATIENTS AND METHODS}

\section{Study design and outcomes}

FORWARD was a randomised, double-blind, dose-finding, placebo-controlled trial. The study design was reported previously. ${ }^{14}$ Patients at 10 centres in seven countries were randomised 1:1:1:1:1 to receive intra-articular sprifermin 100 or $30 \mu \mathrm{g} \mathrm{q} 6 \mathrm{mo}, 100$ or $30 \mu \mathrm{g} \mathrm{q} 12 \mathrm{mo}$ or placebo. Sprifermin was administered in cycles consisting of three injections over three consecutive weeks, q6mo $(0,6,12,18$ months) or q12mo ( 0 and 12 months, with placebo at months 6 and 18) over an 18-month period (online supplemental figure S1).

FORWARD consisted of a primary 2-year analysis period, with an extended 3-year observation period. The primary endpoint was change in TFTJ cartilage thickness in the target knee at year 2, based on quantitative MRI (qMRI, see online supplemental materials). Secondary endpoints included change from baseline in WOMAC scores $(0-100$, where 0 represents no symptoms), $\mathrm{mJSW}$ in the medial and lateral compartments assessed by fixed-flexion radiography, cartilage thickness in medial and lateral femorotibial compartments (MFTC and LFTC) by qMRI, and the incidence and nature of adverse events (AEs) over 2 years. WOMAC assessments, which referenced the last 48 hours, were performed after a pain medication washout of at least 5 half-lives.

Exploratory follow-up for all efficacy and safety endpoints was performed at years 3, 4 and 5. A change in WOMAC pain by $\geq 10$ (of 100) was described as a clinically relevant pain improvement to be perceived by an individual. ${ }^{17}$ The incidence of knee replacements (KRs) in the treated knee was a key exploratory endpoint, added post hoc to the year 5 analyses.

The SAR included all patients with baseline medial or lateral mJSW of 1.5-3.5 mm and a WOMAC pain score of 40-90. Post hoc exploratory analyses were conducted in the SAR, including structural (qMRI-based cartilage thickness and radiographic mJSW), symptomatic (WOMAC scores) and safety endpoints up to year 5 .

See online supplemental materials for details on statistical analyses.

\section{Study participants}

Patients aged 40-85 years with symptomatic radiographic primary femorotibial knee OA, Kellgren-Lawrence Grade (KLG) 2 or 3 , medial $\mathrm{mJSW} \geq 2.5 \mathrm{~mm}$ in the target knee and a history of OA-related pain for 6 months (needing pain medication most days or scoring 4-9 on question A1 of the WOMAC pain index after pain medication washout at screening) were eligible. Exclusion criteria included secondary knee OA, malalignment $>5^{\circ}$ (based on the anatomic axis angle on weight-bearing radiographs), ${ }^{18}$ clinical signs of inflammation in the target knee, planned surgery within 2 years and corticosteroids or hyaluronic acid administration to either knee 6 months before screening. All patients gave written informed consent.

\section{RESULTS}

\section{Patient disposition}

Of the 549 patients randomised (intention to treat (ITT)), 494 $(90 \%)$ were included in the modified ITT (mITT) analysis, 474 $(86 \%)$ completed treatment and the primary 2-year observation period and $442(81 \%)$ and 378 (69\%) completed the 3-year and 5 -year extended follow-up periods, respectively. At baseline, 161 patients (29\%) met the criteria for the SAR (figure 1). The most common reasons for discontinuation were 'withdrawal of consent' $(n=63)$, followed by 'other' $(n=52)$ and 'adverse event' $(n=24)$.

The patients were 40-84 years old, with 69\% being female (online supplemental table S1). Baseline characteristics, including age, sex, body mass index (BMI), mJSW and WOMAC pain were well balanced between study arms. The age, sex, race and BMI of patients in the ITT population and SAR were similar. The SAR, by definition of inclusion, had more severe disease than the ITT population, with a higher proportion of KLG 3 radiographic status (53\% vs 31\%).

\section{Structural endpoints}

The dose-dependent increase in TFTJ cartilage thickness by qMRI with sprifermin relative to placebo detected at year 2 was preserved to year 5 (trend test, $\mathrm{p}<0.001$; figure $2 \mathrm{~A}$ ). In addition, the significant $0.05 \mathrm{~mm}$ adjusted mean increase in TFTJ cartilage thickness change between sprifermin $100 \mu \mathrm{g}$ q6mo and placebo at year 2 was sustained to year $5(0.049 \mathrm{~mm}$; 95\% CI 0.00 to $0.10 ; p=0.015$; figure $2 \mathrm{~B})$. Differences to placebo observed for 


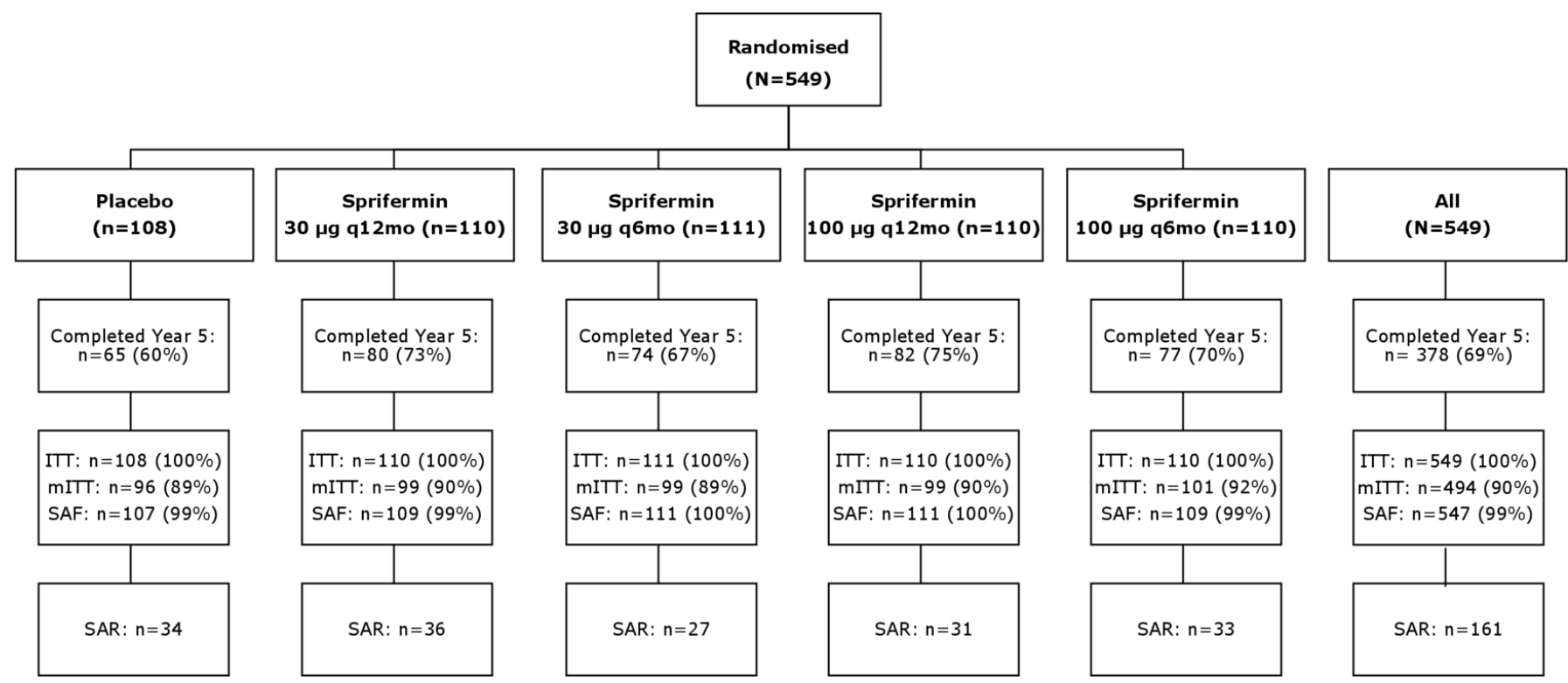

Figure 1 Patient disposition. One patient was randomised to placebo but received sprifermin $30 \mu \mathrm{g}$ at visit 7 and one patient was randomised to sprifermin $30 \mu \mathrm{g} \mathrm{q12mo} \mathrm{but} \mathrm{received} 100 \mu \mathrm{g}$ at visit 3; these patients were included in the $30 \mu \mathrm{g} \mathrm{q} 12 \mathrm{mo}$ and $100 \mu \mathrm{g} \mathrm{q} 12 \mathrm{mo}$ groups, respectively, for the SAF analysis set. ITT, intention to treat; mITT, modified intention to treat; N, total number; n, subgroup sample size; q6mo, every 6 months; q12mo, every 12 months; SAF, safety; SAR, subgroup at risk.

sprifermin $30 \mu \mathrm{g} \mathrm{q} 6 \mathrm{mo}, 100 \mu \mathrm{g} \mathrm{q} 12 \mathrm{mo}$ or $30 \mu \mathrm{g}$ q $12 \mathrm{mo}$ at year 5 were not statistically significant. Results for the SAR at year 5 were consistent with the mITT population, with an adjusted mean difference between the highest sprifermin dose and placebo of $0.06 \mathrm{~mm}$ ( $95 \% \mathrm{CI}-0.02$ to $0.14 ; \mathrm{p}=0.129$; figure $2 \mathrm{C}$ ).

The adjusted mean difference in cartilage thickness change in the MFTC for the highest sprifermin dose compared with placebo was also maintained at approximately $0.05 \mathrm{~mm}$ at each timepoint from years 2-5, although not statistically significant after year $3(95 \% \mathrm{CI}-0.01$ to $0.12 ; \mathrm{p}=0.078$ at year 5; figure 3A). The dose-effect of sprifermin on cartilage thickness change in the LFTC was preserved from years 2 to 5 (trend test, $\mathrm{p}<0.05$ ). The two highest sprifermin dose groups maintained an adjusted mean increase of $0.04 \mathrm{~mm}$ in LFTC compared with placebo to year $5(100 \mu \mathrm{g}$ q12mo: $95 \%$ CI 0.00 to $0.09 ; \mathrm{p}=0.03 ; 100 \mu \mathrm{g} \mathrm{q} 6 \mathrm{mo}: 95 \%$ CI 0.00 to $0.09 ; \mathrm{p}=0.028$; figure $3 \mathrm{~B})$. Results for the SAR at year 5 were consistent with the mITT population, with a mean difference between the sprifermin $100 \mu \mathrm{g}$ q6mo and placebo groups of $0.04 \mathrm{~mm}(95 \% \mathrm{CI}-0.05$ to 0.12$)$ and $0.06 \mathrm{~mm}(95 \% \mathrm{CI}-0.03$ to 0.14 ) in cartilage thickness change in MFTC and LFTC, respectively (figure $3 \mathrm{C}$ and $\mathrm{D}$ ).

No effect of sprifermin on change from baseline in medial mJSW was observed at year 2 or up to year 5 (online supplemental figure S2A, table S2). Yet, a dose response was observed for lateral mJSW at each timepoint $(\mathrm{p}<0.05$ at years $2-5)$. An adjusted mean difference in lateral $\mathrm{mJSW}$ change with sprifermin versus placebo of approximately $0.2 \mathrm{~mm}$ for the $100 \mu \mathrm{g}$ groups was consistently observed over time, but not statistically significant at year $5(100 \mu \mathrm{g}$ q12mo: $95 \% \mathrm{CI}-0.09$ to $0.52 ; \mathrm{p}=0.176 ; 100 \mu \mathrm{g} \mathrm{q} 6 \mathrm{mo}: 95 \% \mathrm{CI}-0.09$ to 0.54 ; $\mathrm{p}=0.167$; online supplemental figure S2B, table S2). For the SAR, a mean difference of $0.16 \mathrm{~mm}(95 \% \mathrm{CI}-0.31$ to 0.62$)$ and $-0.03 \mathrm{~mm}$ (95\% CI -0.48 to 0.42 ) between the highest sprifermin dose and placebo for medial and lateral mJSW, respectively, was observed at year 5 (online supplemental figure S2C,D).

\section{Symptomatic endpoints}

The approximately 20-point (50\%) improvement from baseline in WOMAC pain subscore at year 2 was maintained to year 5 in all cohorts, including placebo (dose response: $p=0.673$; figure 4A, online supplemental table S3). There was no differentiation in WOMAC pain between any sprifermin dose and placebo at year 5 in the ITT cohort; the adjusted mean difference for sprifermin $100 \mu \mathrm{g}$ q6mo versus placebo was -1.06 (CI -9.10 to $6.99 ; p=0.990)$. These results were consistent with WOMAC total scores and WOMAC function subscale scores, which also improved by approximately 20 points $(50 \%)$ from baseline to year 5 in all groups (online supplemental figure S3A, table S3). The adjusted mean difference in WOMAC total for sprifermin $100 \mu \mathrm{g}$ q6mo versus placebo was -1.29 (CI -9.01 to 6.43).

However, post hoc analysis of the SAR identified a significant dose-dependent improvement in WOMAC pain at year 3 that persisted to year 5 (dose response: $p=0.046$; figure 4B). Further, a clinically meaningful numerical difference in WOMAC pain scores between sprifermin $100 \mu \mathrm{g}$ q6mo and placebo at year 3 (adjusted mean -8.58 ; 95\% CI -22.22 to 5.06 ) was sustained to year 5 (adjusted mean $-10.08 ; 95 \% \mathrm{CI}-25.68$ to 5.53 ). In addition, the estimated mean difference in total WOMAC for the SAR between sprifermin $100 \mu \mathrm{g}$ q6mo and placebo was clinically significant and persisted to year $5(-9.50 ; 95 \%$ CI -23.52 to 4.53 ; online supplemental figure S3B).

\section{Safety}

The number of patients who reported AEs over 5 years was similar between the sprifermin groups (96\%-98\%) and placebo (98\%; table 1). AEs were mostly mild or moderate in severity and $79 \%$ were deemed unrelated to treatment. Local AEs in the target knee were reported by 49\%-51\% sprifermin patients and $49 \%$ with placebo (table 1). The greatest number of local related AEs were classified as musculoskeletal and soft tissue disorders, the most common being arthralgia; reported by $9 \%-13 \%$ of 
A

Batch 1

(up to Year 2)
Batch 2 Batch 3 Batch 4 (Year 3) (Year 4) (Year 5)

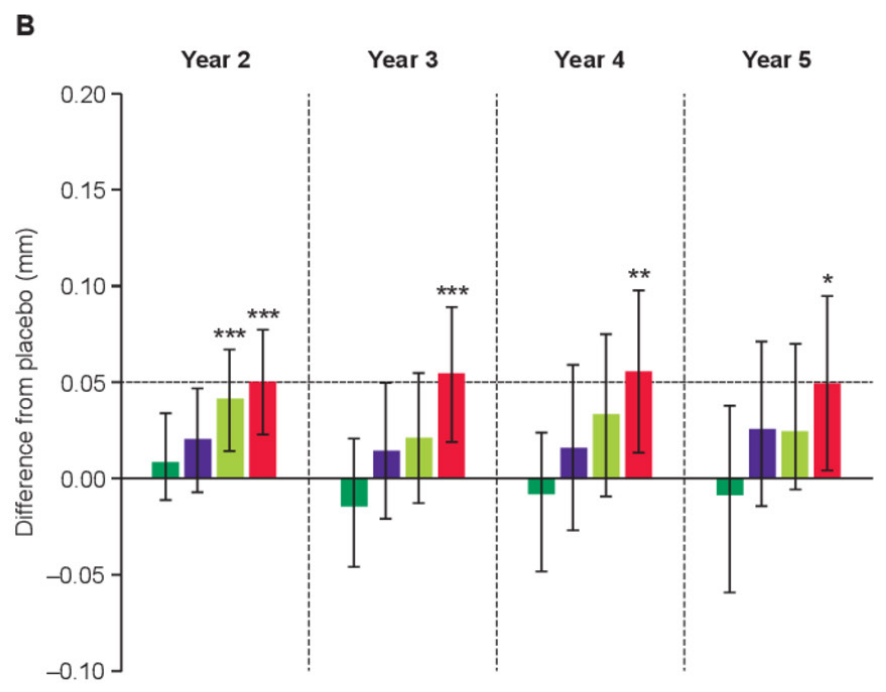

C

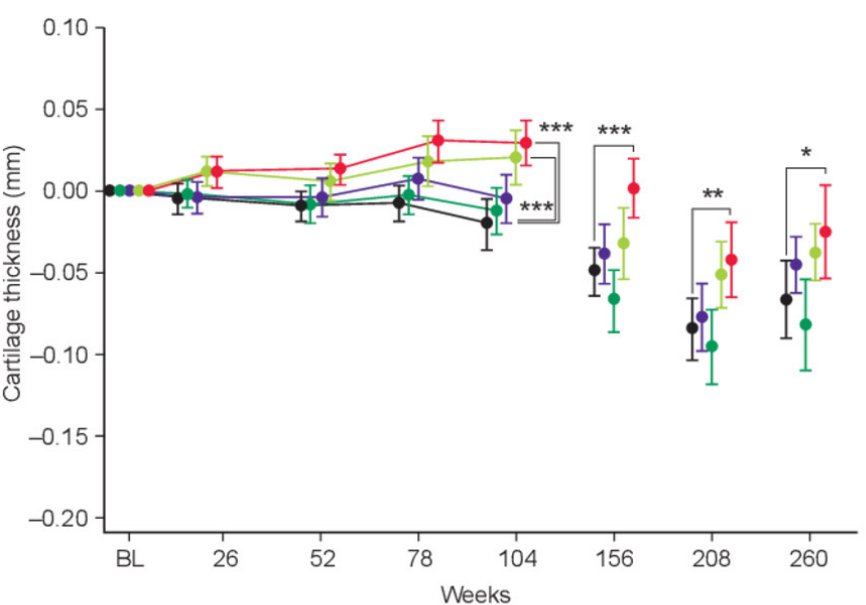

D

Batch 2 Batch 3 Batch 4 (Year 3) (Year 4) (Year 5) $\longmapsto \longmapsto$
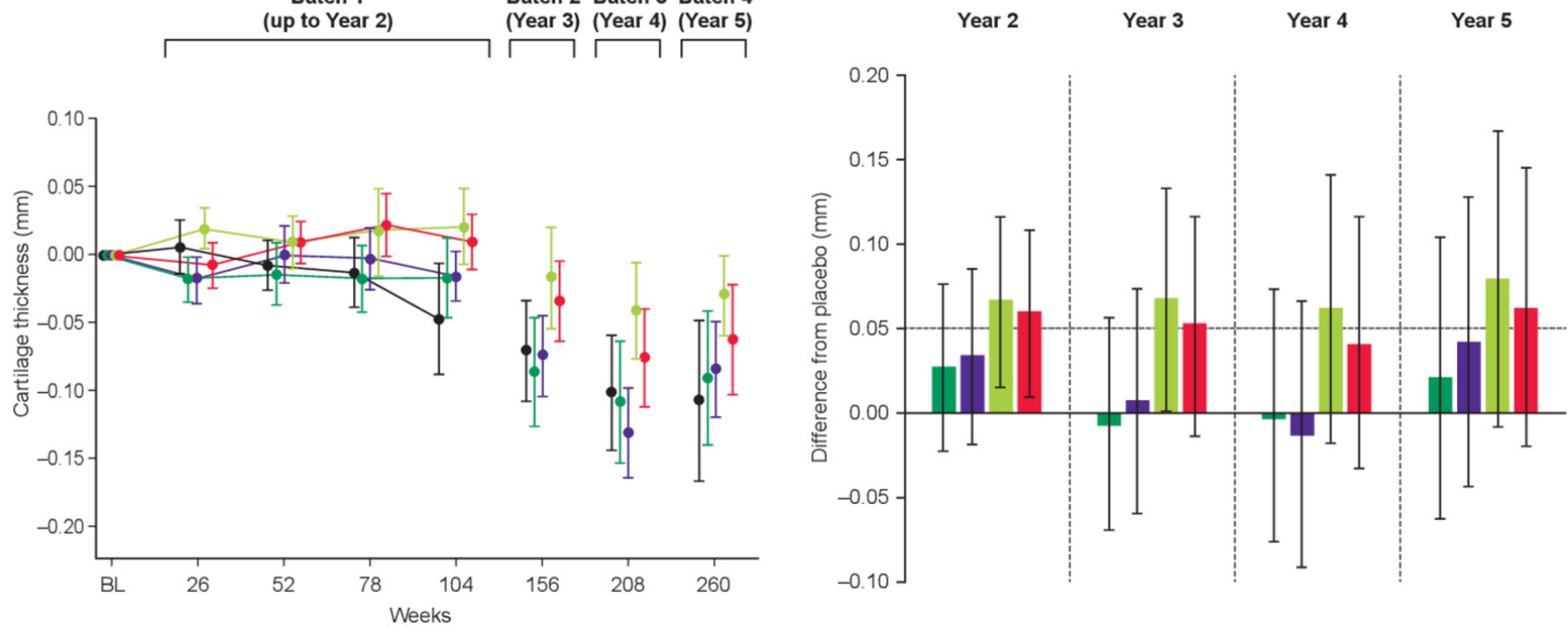

Treatment:

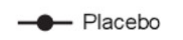

$\longrightarrow$ Sprifermin $100 \mu \mathrm{gg}$ q6mo

$\longrightarrow$ Sprifermin $100 \mu \mathrm{g}$ q12mo

$\longrightarrow$ Sprifermin $30 \mu \mathrm{g}$ q6mo

$\longrightarrow$ Sprifermin $30 \mu \mathrm{g} \mathrm{q} 12 \mathrm{mo}$

Figure 2 Change in TFTJ cartilage thickness up to year 5. (A) Absolute change from baseline in TFTJ cartilage thickness (mm) and (B) difference from placebo in TFTJ cartilage thickness $(\mathrm{mm})$ for the mITT population $(\mathrm{n}=494)$. (C) Absolute change from baseline in TFTJ cartilage thickness (mm) and (D) difference from placebo in TFTJ cartilage thickness (mm) for the SAR ( $n=161)$. Means and 95\% Cls shown. ${ }^{*} p<0.05,{ }^{* *} p<0.005,{ }^{* * *} p<0.001$. BL, baseline; mITT, modified intention to treat; q6mo, every 6 months; q12mo, every 12 months; SAR, subgroup at risk; TFTJ, total femorotibial joint.

patients receiving sprifermin and 9\% receiving placebo. No new systemic or local safety concerns were identified.

A total of 181 patients (33\%) reported serious AEs (SAEs). The incidence of SAEs was similar between the sprifermin (29\%-38\%) and placebo (36\%) groups, and none were considered treatment related by investigators. The most common SAEs were OA and arthralgia. Withdrawals due to AEs were $<10 \%$ in all treatment groups. The majority of these were musculoskeletal and soft tissue disorders. One patient in the placebo group died due to gastric cancer approximately 5 months after the last dose; this was not considered treatment related.

Safety results for the SAR were similar to the ITT population (table 1); there was no obvious difference between the sprifermin and placebo groups and approximately $80 \%$ of AEs were unrelated to treatment. There were more SAEs in the placebo (53\%) than in the sprifermin groups $(31 \%-37 \%)$ for patients in the SAR.

By the end of year 5, a total of 15 patients had partial or total KR of the treated joint (table 1). In the ITT population, the incidence of partial or total KRs in the treated joint was less than $5 \%$ in all groups including placebo $(n=4,4.6 \%)$, with the exception of the sprifermin $30 \mu \mathrm{g}$ q6mo group $(\mathrm{n}=5,5.4 \%)$. No patient in the sprifermin $100 \mu \mathrm{g}$ q6mo group had replacement of the treated knee.

\section{DISCUSSION}

DMOADs that not only alleviate symptoms but also modify structure are an important and unmet clinical need for knee 
A

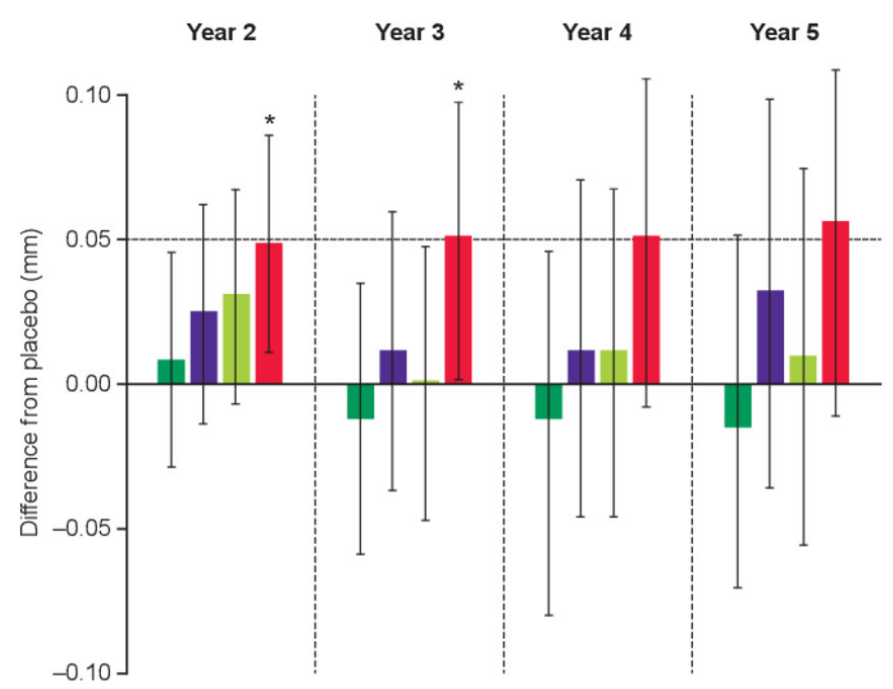

C

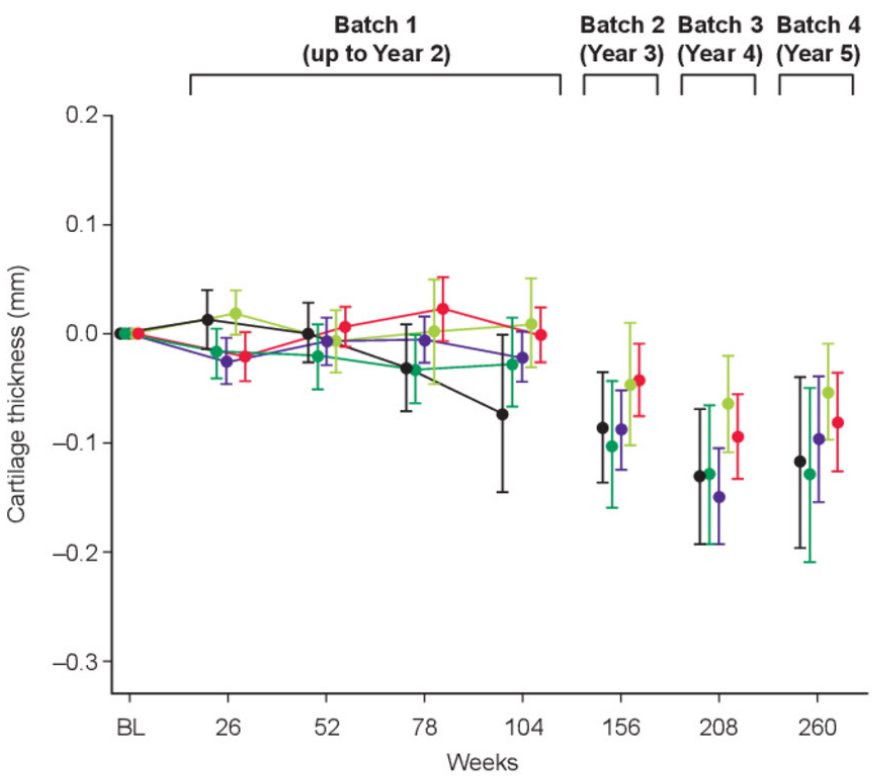

B

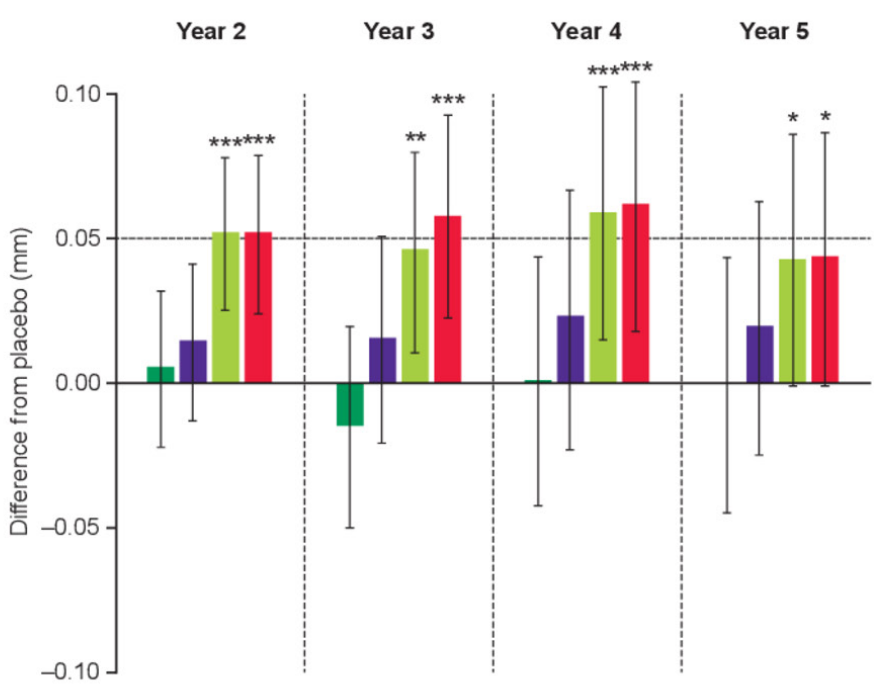

D

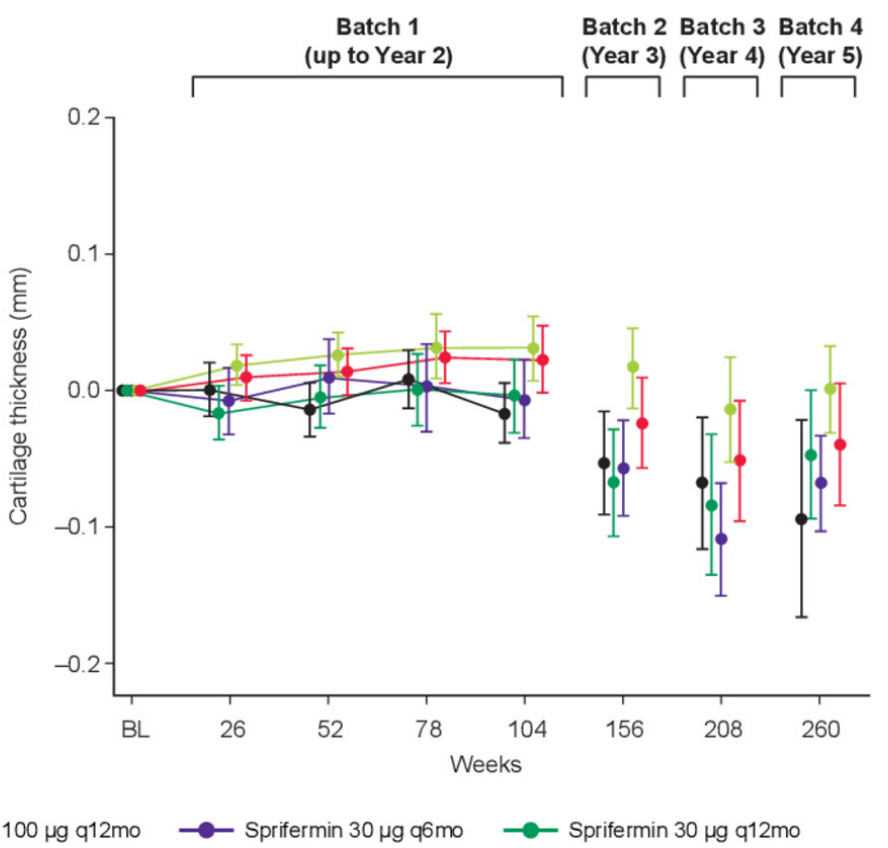

Figure 3 Change in MFTC and LFTC cartilage thickness up to year 5. Difference from placebo in cartilage thickness (mm) in the (A) MFTC and (B) LFTC for the mITT population ( $n=494)$. Absolute change from baseline in (C) MFTC and (D) LFTC cartilage thickness (mm) for the SAR ( $n=161)$. Means and $95 \%$ Cls shown. ${ }^{*} p<0.05,{ }^{* *} p<0.005,{ }^{* *} p<0.001$. BL, baseline; LFTC, lateral femorotibial compartment; MFTC, medial femorotibial compartment; mITT, modified intention to treat; q6mo, every 6 months; q12mo, every 12 months; SAR, subgroup at risk; TFTJ, total femorotibial joint.

OA. Sufficient follow-up in DMOAD trials may be necessary to demonstrate that the structural benefit of a DMOAD is maintained and that structure modification eventually translates into clinical benefit. FORWARD is the longest DMOAD study reported to date. The results of the current analyses show that the efficacy of sprifermin, as measured by both the dose-effect and the mean difference versus placebo in TFTJ cartilage thickness at year 2, was maintained up to year 5, that is, for at least 3.5 years post-active treatment. These findings suggest that intra-articular sprifermin has a sustained effect on articular cartilage modification in patients with knee OA, with no detectable systemic exposure or new safety signals observed up to Year 5.
Evaluation of the type of cartilage gained with sprifermin by biopsy was not possible in FORWARD due to ethical considerations. Preclinical studies in rat models of OA indicated that sprifermin induces the proliferation of hyaline cartilage-producing articular chondrocytes, hyaline extracellular matrix synthesis and cartilage repair. ${ }^{81011}$ Importantly, the cartilage gained with sprifermin treatment up to year 2 did not decline at a faster rate after the end of treatment than the natural cartilage in the placebo group, indicating robust quality and mechanical properties with similar resilience to natural cartilage.

Statistically significant cartilage modification was observed in the LFTC with sprifermin and while the $0.05 \mathrm{~mm}$ net increase in 


\section{A}

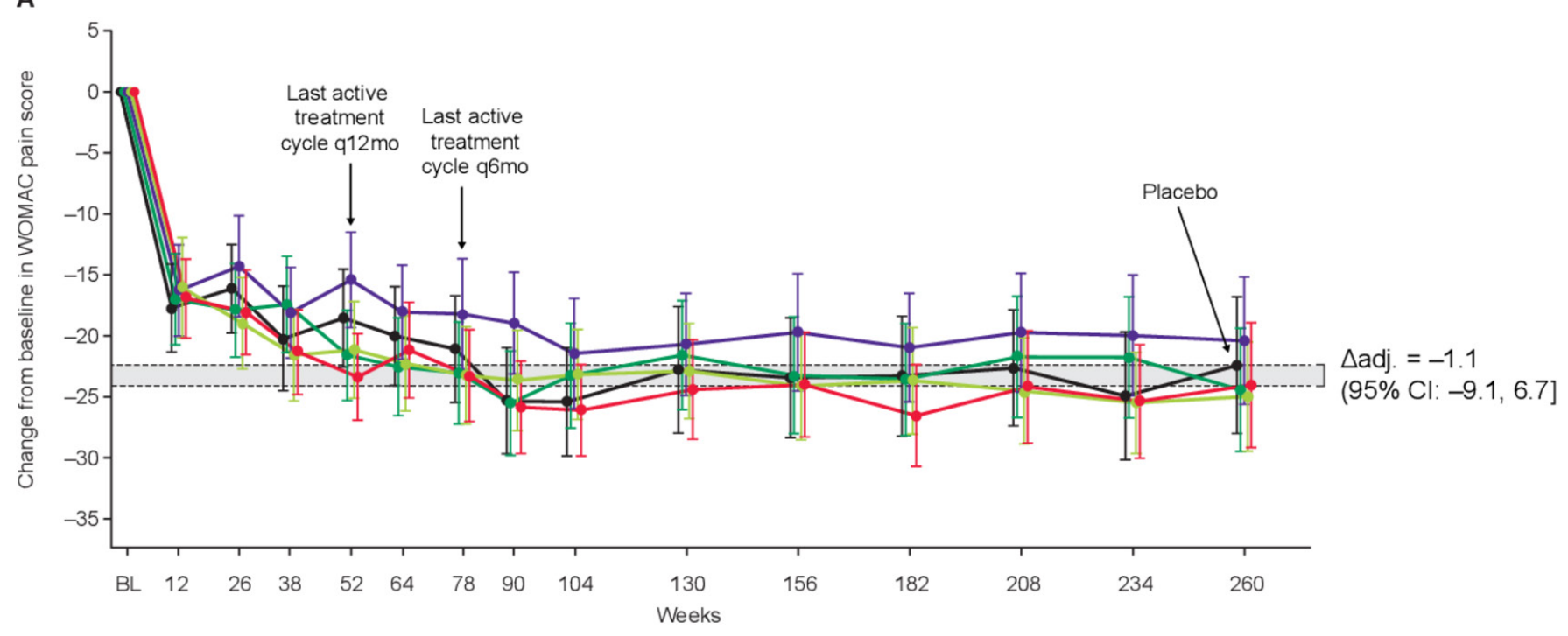

B

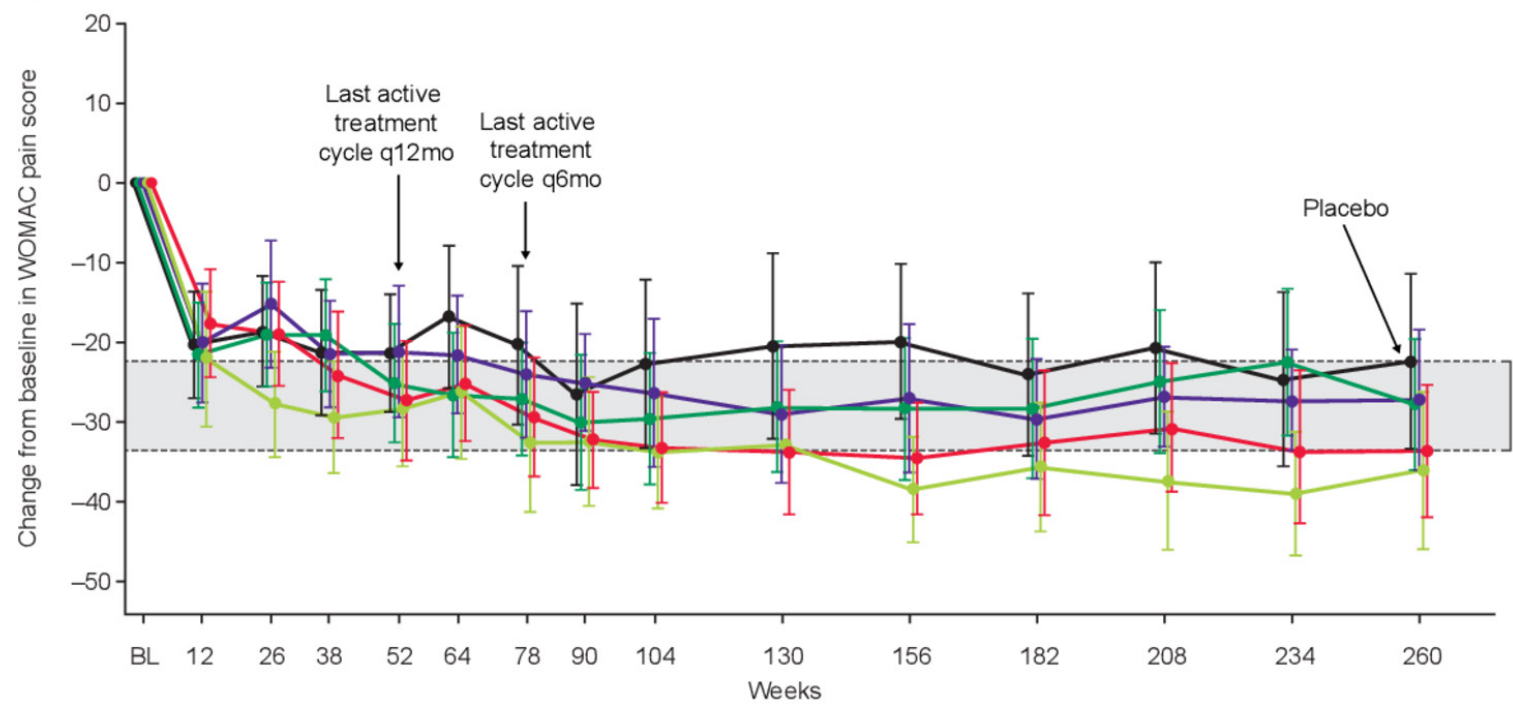

$\Delta$ adj. $=-10.1$

$(95 \% \mathrm{Cl}-25.7,5.5)$

Treatment: $\longrightarrow$ Placebo $\longrightarrow$ Sprifermin $100 \mu \mathrm{g} \mathrm{q6mo} \longrightarrow$ Sprifermin $100 \mu \mathrm{g} \mathrm{q12mo} \longrightarrow$ Sprifermin $30 \mu \mathrm{g}$ q6mo $\longrightarrow$ Sprifermin $30 \mu \mathrm{g} \mathrm{q12mo}$ Figure 4 Change from baseline in WOMAC pain scores up to year 5 in the (A) ITT population $(n=549)$ and (B) SAR ( $n=161)$. Means and 95\% Cls shown. $\triangle$ ajd., adjusted mean difference $(95 \% \mathrm{Cl})$ to placebo at year $5 ; \mathrm{BL}$, baseline; ITT, intention to treat; q6mo, every 6 months; q12mo, every 12 months; SAR, subgroup at risk; WOMAC, Western Ontaria and McMaster Universities Osetoarthritis Index.

MFTC thickness with sprifermin versus placebo was maintained to year 5 , it was not statistically significant. Since the majority of patients in the study had medial disease, less weight load in the lateral compartment (off-loading the joint) may have allowed for a more pronounced effect by sprifermin in the LFTC. Importantly, the medial compartment responded to sprifermin despite continuous weight load.

A large decrease in WOMAC pain of approximately 50\% was observed in all groups, including placebo, and maintained up to year 5. These observations suggest that the contextual effect of intra-articular injection that is known to impact WOMAC outcome in knee OA trials ${ }^{19}$ is maintained over long periods. This is in line with a meta-analysis of 32 randomised trials that showed intra-articular saline injections to significantly improve short-term and long-term knee pain. ${ }^{20}$ There was high variation in WOMAC pain and no statistical differentiation between the highest sprifermin dose and placebo was observed in the ITT cohort at year 5 or previous timepoints. FORWARD was designed to assess structural modification, not symptoms, and as such pain medication was not modified or restricted throughout the study. This may have contributed to the lack of symptomatic differentiation between sprifermin and placebo.

Post hoc analyses identified a subgroup of patients with narrower mJSW $(<3.5 \mathrm{~mm})$ and non-acceptable OA-related knee pain $(>40)$ at baseline: the SAR. ${ }^{16}$ Interestingly, net structural improvement in cartilage thickness relative to placebo was similar in the SAR and overall study population, although the SAR encountered more rapid cartilage loss compared with the latter. This is an important finding, as it is often presumed that DMOADs would be ineffective in patients with more severe 
Table 1 Summary of AEs and KRs in the ITT population $(n=547)$ and SAR at year $5(n=161)$

\begin{tabular}{|c|c|c|c|c|c|c|c|c|c|c|}
\hline \multirow[b]{3}{*}{ Patients, n (\%) } & \multicolumn{5}{|l|}{ ITT } & \multicolumn{5}{|l|}{ SAR } \\
\hline & \multirow{2}{*}{$\begin{array}{l}\text { Placebo } \\
n=107\end{array}$} & \multicolumn{4}{|l|}{ Sprifermin } & \multirow{2}{*}{$\begin{array}{l}\text { Placebo } \\
n=34\end{array}$} & \multicolumn{4}{|c|}{ Sprifermin } \\
\hline & & $\begin{array}{l}30 \mu g \\
q 12 \mathrm{mo} \\
n=109\end{array}$ & $\begin{array}{l}30 \mu \mathrm{g} \mathrm{q} 6 \mathrm{mo} \\
\mathrm{n}=111\end{array}$ & $\begin{array}{l}100 \mu g \\
q 12 \mathrm{mo} \\
\mathrm{n}=111\end{array}$ & $\begin{array}{l}100 \mu \mathrm{g} \\
\mathrm{q} 6 \mathrm{mo} \\
\mathrm{n}=109\end{array}$ & & $\begin{array}{l}30 \mu \mathrm{g} \\
\mathrm{q} 12 \mathrm{mo} \\
\mathrm{n}=36\end{array}$ & $\begin{array}{l}30 \mu g \\
q 6 \mathrm{mo} \\
\mathrm{n}=27\end{array}$ & $\begin{array}{l}100 \mu \mathrm{g} \\
\mathrm{q} 12 \mathrm{mo} \\
\mathrm{n}=31\end{array}$ & $\begin{array}{l}100 \mu \mathrm{g} \\
\mathrm{q} 6 \mathrm{mo} \mathrm{n}=33\end{array}$ \\
\hline All AEs & $105(98.1)$ & $107(98.2)$ & $109(98.2)$ & $107(96.4)$ & $107(98.2)$ & $34(100.0)$ & $35(97.2)$ & $27(100.0)$ & $31(100.0)$ & $33(100.0)$ \\
\hline Local AEs & $52(48.6)$ & $54(49.5)$ & $57(51.4)$ & $54(48.6)$ & $53(48.6)$ & $18(52.9)$ & $17(47.2)$ & $13(48.1)$ & $18(58.1)$ & $12(36.4)$ \\
\hline Systemic AEs & $103(96.3)$ & $104(95.4)$ & $105(94.6)$ & $105(94.6)$ & $106(97.2)$ & $34(100.0)$ & $35(97.2)$ & $27(100.0)$ & $31(100.0)$ & $33(100.0)$ \\
\hline All SAEs & $39(36.4)$ & $35(32.1)$ & $34(30.6)$ & $32(28.8)$ & $41(37.6)$ & $18(52.9)$ & $11(30.6)$ & $10(37.0)$ & $11(35.5)$ & $12(36.4)$ \\
\hline Local SAEs & $5(4.7)$ & $4(3.7)$ & $6(5.4)$ & $3(2.7)$ & $1(0.9)$ & $4(11.8)$ & $1(2.8)$ & $0(0.0)$ & $0(0.0)$ & $0(0.0)$ \\
\hline SAEs leading to death & $1(0.9)$ & $0(0.0)$ & $0(0.0)$ & $0(0.0)$ & $0(0.0)$ & $1(2.9)$ & $0(0.0)$ & $0(0.0)$ & $0(0.0)$ & $0(0.0)$ \\
\hline $\mathrm{KRs}^{*}$ & $4(4.6)$ & $4(4.0)$ & $5(5.4)$ & $2(2.0)$ & $0(0.0)$ & $3(8.8)$ & $1(2.8)$ & $1(3.7)$ & $0(0.0)$ & $0(0.0)$ \\
\hline
\end{tabular}

${ }^{*}$ The incidence of partial or total replacement of the treated knee at year 5, presented as a percentage of patients who completed year 2 per group (placebo, $\mathrm{N}=87 ; 30 \mu \mathrm{g}$ q12mo, N=99; $30 \mu \mathrm{g} \mathrm{q6mo,} \mathrm{N=92;100 \mu g} \mathrm{q12mo,} \mathrm{N=98;} 100 \mu \mathrm{g} \mathrm{q6mo,} \mathrm{N}=96)$.

AEs, adverse events; ITT, intention to treat; KRs, knee replacements; q6mo, every 6 months; q12mo, every 12 months; SAEs, serious adverse events; SAR, subgroup at risk.

disease at baseline and in those with faster structural progression. The current results, however, demonstrate that structural modification by sprifermin is at least as strong in more severe $\mathrm{OA}$ as in milder OA.

The overall study population was relatively heterogeneous due to the inclusion criteria allowing a population with diverse levels of baseline pain and structural status. A shift in pain reporting between screening (WOMAC sub-question A1) and baseline (sub-questions A1-A5) was observed, and a relatively high proportion of patients (32\%) had a baseline WOMAC pain score of $<40$. Such heterogeneous cohorts may be less likely to differentiate DMOAD responses on pain from placebo due to a floor effect that contributes to lower average scores across the study population. ${ }^{16}$ Patients were selected with different amounts of cartilage thickness at baseline in order to maximise the likelihood of observing structural changes. As a result, many patients $(38 \%)$ had a baseline medial mJSW of $>4.0 \mathrm{~mm}$ that approached the JSW of healthy individuals. It is likely that more time is required for these patients to show translation of structural improvement to symptomatic benefit versus placebo.

Importantly, and in contrast with the full ITT cohort, the potential clinically meaningful improvements in pain with the highest sprifermin dose versus placebo observed at year 3 were sustained to year 5 in the SAR. The pain benefit of sprifermin in this subgroup required 2-3 years to become clinically relevant, in line with the hypothesis that this occurs subsequent to structural modification of cartilage thickness. These results are plausible, as patients with less mJSW, lower cartilage thickness and more pain at baseline are more likely to symptomatically benefit from cartilage structural modification than those with only mild disease. The findings also show that relatively long observation intervals may be required to demonstrate the translation of structural modification into symptomatic benefit.

The limitations of this study include that the year 5 analyses were exploratory, and the SAR was identified post hoc and included a modest proportion of patients. Conclusions regarding sprifermin's symptomatic effects are limited by the lack of control/modification of analgesic medication during the study (as per protocol) and the enrolment of patients with limited pain at baseline. No techniques were applied to identify valid pain reporters or limit the placebo effect on pain. Rescue medications and changes in physical activity were not analysed, which could have helped put into context the lack of separation in symptomatic endpoints between the sprifermin and placebo groups. Finally, MRI reading approaches regarding blinding to the sequence of image acquisition differed between time points, although differences between sprifermin-treated and placebo-treated patients at each time point were unaffected by this.

In conclusion, these findings suggest potential long-lasting cartilage structural modification with sprifermin treatment among patients with knee OA. Symptomatic improvements with sprifermin versus placebo were observed in an SAR of structural and symptomatic progression. The FORWARD trial had the longest follow-up time for DMOAD efficacy using quantitative imaging and provides an exemplar for the feasibility of long-term OA trials. Enrichment of patient populations with higher likelihood of structural and symptomatic progression may increase the chance of successful DMOAD development.

\section{Author affiliations \\ ${ }^{1}$ Institute of Anatomy and Cell Biology, Paracelsus Medical University Salzburg and Nuremberg, Salzburg, Austria \\ ${ }^{2}$ Ludwig Boltzmann Institute for Arthritis and Rehabilitation, Paracelsus Medical University, Salzburg, Austria \\ ${ }^{3}$ Chondrometrics GmbH, Ainring, Germany \\ ${ }^{4}$ University of Maryland School of Medicine, Baltimore, Maryland, USA \\ ${ }^{5}$ Merck KGaA, Darmstadt, Hessen, Germany \\ ${ }^{6}$ EMD Serono Research and Development Institute, Inc, Billerica, Massachusetts, USA; an affiliate of Merck KGaA, Darmstadt, Germany \\ ${ }^{7}$ Nordic Bioscience, Herlev, Denmark \\ ${ }^{8}$ Rheumatology, Leeds Teaching Hospitals NHS Trust, Leeds, UK}

Acknowledgements Medical writing and editorial support were provided by Bioscript Science, who received payment for their work. The manuscript was written in accordance with Good Publication Practice (GPP3) guidelines (http://www.ismpp. org/gpp3). PGC is supported in part by the UK National Institute for Health Research (NIHR) Leeds Biomedical Research Centre. The authors thank those who participated in the FORWARD study and their families, the FORWARD investigators and research staff, including Jeffery Kraines, MD, for his valuable advice and support related to the FORWARD study, as well as NBCD (Nordic Bioscience Clinical Development) and Chondrometrics $\mathrm{GmbH}$. The authors would also like to thank Wolfgang Wirth (Chondrometrics $\mathrm{GmbH}$, Germany) for valuable input while reviewing the final version of the manuscript.

Contributors The study was funded by Merck KGaA, Darmstadt, Germany, and Billerica, Massachusetts, USA, who was involved in the study design, the collection, analysis and interpretation of data, and the development of the manuscript. All authors were involved in the analysis and interpretation of data, the review and approval of the manuscript, and had final responsibility for the decision to submit for publication. FE, MH, HG, FM, ARB and JRA were involved in the study design and 
concept. Medical writing and editorial support were provided by Bioscript Stirling and funded by Merck KGaA, Darmstadt, Germany.

Funding The study was funded by Merck KGaA, Darmstadt, Germany, and Billerica, Massachusetts, USA.

Disclaimer The views expressed are those of the authors and not necessarily those of the NIHR or the Department of Health and Social Care.

Competing interests $\mathrm{FE}$ is an employee and co-owner of Chondrometrics $\mathrm{GmbH}$ and reported receiving grants and consulting fees from Merck KGaA, Darmstadt, Germany, Samumed LLC, AbbVie, Bioclinica, Orthotrophix, Kolon TissueGene, Servier, Roche, Galapagos, Novartis, ICM and Healthlink. MH reports being the president of Rheumcon and receiving consulting fees from Bone Therapeutics, Bristol-Myers Squibb, Eli Lilly, Galapagos, IBSA Insititut Biotechniq SA, Novartis Pharma AG, Pfizer, Samumed LLC, Theralogix LLC and Kolon TissueGene. HG, BD, OG, CL and MM are employees of Merck KGaA, Darmstadt, Germany. FM and VO are employees of EMD Serono (a business of Merck KGaA, Darmstadt, Germany). ARB, IB and JRA are employees and shareholders in Nordic Bioscience. PGC has received consultancy or speaker's bureau fees from AbbVie, AstraZeneca, BMS, EMD Serono (a business of Merck KGaA, Darmstadt, Germany), Flexion Therapeutics, Galapagos, Kolon TissueGene, Novartis, Pfizer, Samumed and Stryker.

Patient consent for publication Not required.

Ethics approval The study was performed in accordance with the ethical principles of the Declaration of Helsinki.

Provenance and peer review Not commissioned; externally peer reviewed.

Data availability statement Data are available upon reasonable request. Any requests for data by qualified scientific and medical researchers for legitimate research purposes will be subject to Merck KGaA, Darmstadt, Germany's Data Sharing Policy. All requests should be submitted in writing to Merck KGaA, Darmstadt, Germany, via their data sharing portal. When Merck KGaA, Darmstadt, Germany, has a co-research, co-development, or co-marketing or co-promotion agreement, or when the product has been out licensed, the responsibility for disclosure might be dependent on the agreement between parties. Under these circumstances, Merck KGaA, Darmstadt, Germany, will endeavour to gain agreement to share data in response to requests.

Supplemental material This content has been supplied by the author(s). It has not been vetted by BMJ Publishing Group Limited (BMJ) and may not have been peer-reviewed. Any opinions or recommendations discussed are solely those of the author(s) and are not endorsed by BMJ. BMJ disclaims all liability and responsibility arising from any reliance placed on the content. Where the content includes any translated material, BMJ does not warrant the accuracy and reliability of the translations (including but not limited to local regulations, clinical guidelines, terminology, drug names and drug dosages), and is not responsible for any error and/or omissions arising from translation and adaptation or otherwise.

Open access This is an open access article distributed in accordance with the Creative Commons Attribution Non Commercial (CC BY-NC 4.0) license, which permits others to distribute, remix, adapt, build upon this work non-commercially, and license their derivative works on different terms, provided the original work is properly cited, appropriate credit is given, any changes made indicated, and the use is non-commercial. See: http://creativecommons.org/licenses/by-nc/4.0/.

ORCID iDs

Felix Eckstein http://orcid.org/0000-0002-2014-8278

Philip G Conaghan http://orcid.org/0000-0002-3478-5665

\section{REFERENCES}

1 Bannuru RR, Osani MC, Vaysbrot EE, et al. OARSI guidelines for the non-surgical management of knee, hip, and polyarticular osteoarthritis. Osteoarthritis Cartilage 2019;27:1578-89.

2 Kolasinski SL, Neogi T, Hochberg MC, et al. 2019 American College of Rheumatology/ Arthritis Foundation guideline for the management of osteoarthritis of the hand, hip, and knee. Arthritis Rheumatol 2020;72:220-33.

3 Martel-Pelletier J, Barr AJ, Cicuttini FM, et al. Osteoarthritis. Nat Rev Dis Primers 2016;2:16072.

4 Hermann W, Lambova S, Muller-Ladner U. Current treatment options for osteoarthritis. Curr Rheumatol Rev 2018;14:108-16.

5 Karsdal MA, Michaelis M, Ladel C, et al. Disease-Modifying treatments for osteoarthritis (DMOADs) of the knee and hip: lessons learned from failures and opportunities for the future. Osteoarthritis Cartilage 2016:24:2013-21.

6 Ghouri A, Conaghan PG. Update on novel pharmacological therapies for osteoarthritis. Ther Adv Musculoskelet Dis 2019:11:1759720X1986449.

7 Brandt KD, Mazzuca SA. Lessons learned from nine clinical trials of disease-modifying osteoarthritis drugs. Arthritis Rheum 2005:52:3349-59.

8 Gigout A, Guehring H, Froemel D, et al. Sprifermin (rhFGF18) enables proliferation of chondrocytes producing a hyaline cartilage matrix. Osteoarthritis Cartilage 2017;25:1858-67.

9 Reker D, Kjelgaard-Petersen CF, Siebuhr AS, et al. Sprifermin (rhFGF18) modulates extracellular matrix turnover in cartilage explants ex vivo. J Trans/ Med 2017;15:250.

10 Sennett ML, Meloni GR, Farran AJE, et al. Sprifermin treatment enhances cartilage integration in an in vitro repair model. J Orthop Res 2018;36:2648-56.

11 Moore EE, Bendele AM, Thompson DL, et al. Fibroblast growth factor-18 stimulates chondrogenesis and cartilage repair in a rat model of injury-induced osteoarthritis. Osteoarthritis Cartilage 2005;13:623-31.

12 Dahlberg LE, Aydemir A, Muurahainen N, et al. A first-in-human, double-blind, randomised, placebo-controlled, dose ascending study of intra-articular rhFGF18 (sprifermin) in patients with advanced knee osteoarthritis. Clin Exp Rheumatol 2016;34:445-50

13 Lohmander LS, Hellot S, Dreher D, et al. Intraarticular sprifermin (recombinant human fibroblast growth factor 18) in knee osteoarthritis: a randomized, double-blind, placebo-controlled trial. Arthritis Rheumatol 2014:66:1820-31.

14 Hochberg MC, Guermazi A, Guehring H, et al. Effect of intra-articular Sprifermin vs placebo on femorotibial joint cartilage thickness in patients with osteoarthritis: the forward randomized clinical trial. JAMA 2019;322:1360-70.

15 Bowes M, Guillard G, Brett A, et al. OP0164 optimizing recruitment criteria for an osteoarthritis structure modification trial: data from the OAI. Ann Rheum Dis 2017;76:119-20.

16 Gühring H, Moreau F, Daelken B, et al. The effects of sprifermin on symptoms and structure in a subgroup at risk of progression in the forward knee osteoarthritis trial. Semin Arthritis Rheum 2021.

17 Bellamy N, Hochberg M, Tubach F, et al. Development of multinational definitions of minimal clinically important improvement and patient acceptable symptomatic state in osteoarthritis. Arthritis Care Res 2015;67:972-80.

18 Kraus VB, Vail TP, Worrell T, et al. A comparative assessment of alignment angle of the knee by radiographic and physical examination methods. Arthritis Rheum 2005:52:1730-5.

19 Zhang W. The powerful placebo effect in osteoarthritis. Clin Exp Rheumatol 2019;37 Suppl 120:118-23.

20 Altman RD, Devji T, Bhandari M, et al. Clinical benefit of intra-articular saline as a comparator in clinical trials of knee osteoarthritis treatments: a systematic review and meta-analysis of randomized trials. Semin Arthritis Rheum 2016;46:151-9. 